\title{
Der öffentliche Auftrag der Soziologie ${ }^{1}$
}

Vor ein paar Jahren, bei einer internationalen wissenschaftlichen Konferenz ungefähr zu der Zeit, als Michael Burawoy seinen Aufruf für eine »öffentliche Soziologie « formulierte, ${ }^{2}$ kam mir der Gedanke, dass noch nie zuvor in der Geschichte so viele Menschen so gut darin ausgebildet waren wie heute, das soziale Leben zu erklären und zu verstehen. Doch die mächtigsten Politiker, die diese soziologisch hochgebildete Generation - meine Generation - hervorgebracht hatte, waren George W. Bush und Dick Cheney, damals gerade wiedergewählt und mit dem Auftrag versehen, die führende Demokratie der Welt vier weitere Jahre lang zu regieren. Seit dieser Zeit hat mich die Diskrepanz zwischen dem fortschreitenden Verfall von Politik und Ökonomie der Vereinigten Staaten einerseits und der akademischen Exzellenz von sozialwissenschaftlichen Fakultäten wie Harvard und Berkeley andererseits nicht mehr losgelassen. Wozu war all diese Brillanz am Ende gut? Manchmal habe ich amerikanische Kollegen in privater Runde, sozusagen am Abend nach der Arbeit, gefragt, was sie tun, um sich Gehör zu verschaffen, wenn ihrer Öffentlichkeit weisgemacht wird, dass Kriege wie die im Irak oder in Afghanistan ein demokratisches »nation-building « zum Ziel hätten - ob dies nicht ein Thema sei, zu dem die Sozialwissenschaften etwas beisteuern könnten. Die Antwort war stets ein resigniertes Schweigen: Warum sich die Mühe machen, es hört ja sowieso niemand zu.

\section{Soziologie und Öffentlichkeit: Ein Problem der Nachfrage?}

Hat die Soziologie diesseits des Atlantiks ein öffentliches Publikum, bei weniger dramatischen Themen? Ich habe dazu keine empirische Untersuchung durchgeführt, doch mein Eindruck als teilnehmender Beobachter ist: wenn überhaupt, dann nur ein sehr kleines. Ich lese regelmäßig die Wissenschaftsrubriken unserer führenden Tageszeitungen, aus Gründen, die mit diesem Vortrag nichts zu tun haben, und stelle fest, dass über Psychologie, Hirnforschung und Evolutionsbiologie weitaus mehr berichtet wird als über Soziologie. Auch die Wirtschaftswissenschaften sind vertreten, vor allem ihre jüngsten Ableger, die Verhaltensökonomik und die Neuroökonomie. Das eigentliche Revier der Ökonomen sind natürlich der Wirtschafts- und

1 Deutsche Übersetzung eines Beitrags zu einer Konferenz des Social Science Research Councils der Vereinigten Staaten und des Wissenschaftszentrums Berlin für Sozialforschung zum Thema The Public Mission of the Social Sciences and Humanities: Transformation and Renewal, 16. und 17. September 2011.

2 Burawoy 2005. 
der Politikteil, die beide erheblich mehr Gewicht haben als der Wissenschaftsteil und in denen die Soziologie, von ein paar Ausnahmen abgesehen, völlig fehlt.

Warum ist das so? Ein denkbarer Grund ist, dass die Soziologie weniger als andere Disziplinen dem entspricht, was die meisten unter Wissenschaft verstehen und weswegen sie sich für wissenschaftliche Themen interessieren. Was ein Laienpublikum an Psychologie, Verhaltensökonomik, Evolutionsbiologie und ähnlichen Disziplinen fasziniert, ist, dass sie vorgeben, verborgene Ursachen von Handlungen aufdekken zu können, von denen wir normalerweise glauben wollen oder sollen, dass sie von bewussten Gründen geleitet sind - Ursachen, die insgeheim bestimmen, was wir tun, ohne dass wir es merken. Ein Beispiel für diese Art von Forschung, die im deutschen Wissenschaftsjournalismus zuletzt immer wieder Aufmerksamkeit erregt hat, ist, was ich das T-Shirt-Schnüffel-Experiment nennen möchte. Dieses soll zeigen, dass Frauen den Geruch von Männern bevorzugen, deren genetischer Aufbau dem ihren in dem Sinne am besten entspricht, als er bei gemeinsamer Fortpflanzung gesünderen Nachwuchs erwarten lässt. ${ }^{3}$ Ein anderes, ständig wiederkehrendes Motiv ist sexuelle Untreue bei monogamen Vogelarten; wie gerne berichtet wird, ist diese häufiger als man denkt, wobei sich Weibchen angeblich umso häufiger mit anderen als mit ihren lebenslangen Partnern paaren, wenn schon ihre Väter häufig untreu waren, was aus irgendwelchen komplizierten evolutionsbiologischen Gründen mit der »Fitness « der jeweils zu erwartenden Nachkommen zusammenhängen soll. ${ }^{4}$

Nicht, dass es überhaupt kein Interesse an soziologischer Forschung gäbe. Untersuchungen über sexuelles Verhalten, da bin ich mir sicher, fänden weithin Beachtung. Doch mit Sexualität befasst sich die Soziologie ja nicht mehr. ${ }^{5}$ Stattdessen beschäftigt sie sich mit Gender, und tatsächlich werden Forschungsergebnisse zum Kampf der Geschlechter in all seinen Varianten - Aufteilung der Hausarbeit, Lohnungleichheit, Alltag alleinerziehender Mütter - eifrig berichtet und, so nehme ich an, ebenso eifrig gelesen. Ausschlaggebend hierfür ist wohl ein hoher Konversationsund Unterhaltungswert, der auch demoskopischen Befindlichkeitsumfragen aller Art anhängt. Ebenfalls rezipiert, wenn auch eher zufällig, werden Untersuchungen zum Schul- und Bildungssystem, zur sozialen Mobilität und Elitenbildung oder zur Einwanderung und ihren Licht- und Schattenseiten. Hier könnte man von Sozialberichterstattung in einem weiten Sinn sprechen, die die Neugier der Leser anspricht und deren Kenntnisnahme als Ausweis staatsbürgerlicher Allgemeinbildung dienen kann. Weniger konversationsgeeignet sind freilich die Theorien, mit denen die be-

3 »Liegt Freundschaft in den Genen? Ob sich Menschen untereinander verlieben, hängt davon ab, ob ihr Erbgut zusammen passt. Mit der Wahl von Freunden ist es ähnlich «, Die Welt, 19.01.2011, S. 22.

4 »Vaterschaftstest im Nistkasten - Warum sich Fremdgehen lohnen kann«, BIOMAX, Max-Planck-Forschung, Ausgabe 26, Herbst 2010; "Seitensprung in der Voliere «, Süddeutsche Zeitung, 15.06.2011, S.16; »Wie der Opa, so die Töchter", Laborjournal, 01.09.2011, S. 56-57; »Testosteron-Vögelchen treiben es gar zu bunt «, Ludwigsburger Kreiszeitung, 24.10.2011, S. 15.

5 Eine Ausnahme, die die Regel bestätigt, ist Jean-Claude Kaufmanns »Sex@mour. Wie das Internet unser Liebesleben verändert « (Kaufmann 2011). 
richteten Befunde zu erklären wären, und von ihnen wird denn auch nur selten Notiz genommen.

Theorie ist allerdings nicht immer verpönt. Das meistverkaufte deutsche Sachbuch des Jahrzehnts, wenn nicht sogar der deutschen Nachkriegszeit, ist Thilo Sarrazins 2010 erschienenes Deutschland schafft sich ab. ${ }^{6}$ Der Autor, SPD-Politiker, ist ein prominenter sogenannter »Islamkritiker «. Das Fazit seines Buchs ist, knapp zusammengefasst, dass Einwanderung aus islamischen Ländern in Verbindung mit der niedrigen Geburtenrate der gut ausgebildeten deutschen Mittelschicht die Qualität des deutschen Genpools verschlechtert und den durchschnittlichen Intelligenzquotienten der Bevölkerung herunterdrückt, was langfristig die Wettbewerbsfähigkeit der deutschen Wirtschaft beeinträchtigen müsse. Sarrazin ist studierter Ökonom, doch das Buch stützt sich weitgehend auf Forschungsergebnisse aus Psychologie und Demografie und wagt sich auch auf soziologisches Terrain, etwa wenn es um den Zusammenhang zwischen Intelligenz und Religion einerseits und der sozioökonomischen Stellung andererseits geht. Es ist wohl nicht übertrieben, wenn man das Buch als neo-eugenisches Manifest bezeichnet, mit einer biologistischen Weltsicht und stark rassistischen Untertönen (etwa wenn von Inzest in arabischen Großfamilien die Rede ist, der die Intelligenz ihrer Nachkommen vermindere), eingebettet in eine Effizienztheorie von Wirtschaft und Gesellschaft in einer globalisierten Ökonomie.

Der Fall Sarrazin erscheint in mehrfacher Hinsicht aufschlussreich. Er zeigt, dass es in Deutschland durchaus ein Publikum - und kein kleines - für Bücher mit sozialwissenschaftlichem Inhalt gibt, selbst wenn diese gespickt sind mit Statistiken und langen Diskussionen wissenschaftlicher Zeitschriftenartikel. ${ }^{7}$ Warum meidet die akademische Soziologie diesen Markt? Die seriöse Auseinandersetzung mit Sarrazin blieb im Wesentlichen den Journalisten der Qualitätszeitungen überlassen, insbesondere der Frankfurter Allgemeinen Zeitung und der Süddeutschen Zeitung. Darüber hinaus meldeten sich ein paar Entwicklungspsychologen mit der Erkenntnis zu Wort, dass Intelligenz nicht ausschließlich eine Frage der Vererbung sei, und Religionswissenschaftler teilten mit, dass es viele Islame innerhalb des Islams gebe. Von Soziologen aber kam wenig bis nichts - allerdings kam auch kaum jemand auf die Idee, sie zu fragen. ${ }^{8}$ Noch weniger kam von Ökonomen, denen der offenkundige Ökonomismus des Buchs freilich auch kaum unsympathisch gewesen sein dürfte.

Warum ist die Soziologie in öffentlichen Debatten dieser Art so wenig präsent? Eine mögliche Antwort ist: Ihre Betreiber ahnen, dass sie schlechte Karten hat. Für

6 Sarrazin 2010.

7 Vom Flughafen zum Konferenzort wurde der Autor von einem jener (West-)Berliner Taxifahrer gefahren, deren Meinungsfreudigkeit vielen Journalisten immer wieder zum Segen gereicht. Er hatte während seiner Wartezeit Sarrazin studiert und war bis in die Mitte des Buchs vorgedrungen, wo er, bevor er das Buch auf den Beifahrersitz legte, gewissenhaft ein Lesezeichen unterbrachte.

8 Dass und wie Sarrazin und seine Leser selber Anstoß zu interessanten gesellschaftswissenschaftlichen Reflexionen geben können, hat kürzlich eine Literaturwissenschaftlerin gezeigt (Lange 2011). 
die meisten Menschen ist Wissenschaft die Entdeckung allgemeiner Gesetzmäßigkeiten, aus denen sich sparsame kausale Erklärungen ableiten lassen, die in technisches Know-how oder moralische Rechtfertigungen übersetzt werden können - oder idealiter in beides zugleich, wie im Fall des homo oeconomicus, in der Evolutionsbiologie (»selbst die Vögel tun es, und mit guten Gründen«) oder in der neurologischen Diskussion um die Existenz eines »freien Willens «. Die Soziologie dagegen befasst sich mit historisch einzigartigen Situationen, in denen überdies stets mehr als ein kausaler Faktor wirksam ist; und wenn sie es überhaupt wagt, Vorhersagen zu treffen, dann muss sie diese, wenn sie ehrlich ist, vielfältig konditionieren. Im Gegensatz zur Psychologie und zu den Naturwissenschaften kann die Soziologie kaum versprechen, geheime materielle Kräfte aufzuspüren, die hinter dem sichtbaren Weltgeschehen stehen und es bestimmen. Ihre Erkenntnisse muss sie mit der eindringlichen Warnung versehen, dass sie nicht übergeneralisiert werden dürfen, und mit vielfältigen Hinweisen auf soziale, ökonomische und kulturelle Kontextbedingungen, die die jeweils wirkenden Kausalbeziehungen beeinflussen und modifizieren. Einige von uns glauben, dies liege daran, dass die Soziologie noch immer eine relativ junge Disziplin ist, die erst noch wirklich "wissenschaftlich « werden muss. Andere dagegen denken, wie ich auch, dass der Zustand des soziologischen Wissens der speziellen Ontologie der sozialen Welt geschuldet ist. Wie dem auch sein mag - die Soziologie in ihrer heute praktizierten Form kann dem szientistischen Standardmodell von Wissenschaft nicht gerecht werden und wird deshalb von einer Öffentlichkeit, die an dieses Modell glaubt, ${ }^{9}$ nur allzu oft als enttäuschend empfunden.

Ein weiteres Problem, mit der die Soziologie zumindest hierzulande zu kämpfen hat, ist ihr Bild in der Öffentlichkeit, das noch aus den 1970er Jahren stammt. Soziologie gilt als "weich ", nicht nur im wissenschaftlichen Sinn, sondern auch politisch, und Weichheit ist in harten Zeiten außer Mode. Der Soziologe steht im Verdacht übertriebener Empathie mit seinen Forschungsobjekten, bei denen es sich häufig um Randgruppen wie Langzeitarbeitslose, Kriminelle und »Parallelgesellschaften « jeder Art handelt - oder ganz allgemein um die in einer immer anspruchsvoller gewordenen »Leistungsgesellschaft « abgehängte Überschussbevölkerung. Oft »erklärt « die Soziologie deren Lebensweise, indem sie den »Sinn « expliziert, den diese ihrem Handeln selbst zusprechen. Im Deutschen sprechen wir mit Max Weber von » verstehender Soziologie «. Doch Verstehen wird nicht immer geschätzt in einer Gesellschaft, in der ein bekanntes Sprichwort lautet: "Alles verstehen heißt alles verzeihen «. Eine Soziologie, die es auf sich nimmt, das Handeln der Menschen zu erklären, indem sie deutlich macht, welchen Sinn sie diesem beimessen, kann leicht als wissenschaftlich getarntes Advokaten- oder gar »Gutmenschentum « er-

9 Ein Glaube, der im Übrigen von nicht wenigen Sozialwissenschaftlern geteilt wird, mit der Folge immer neuer Demutsbekundungen gegenüber den Naturwissenschaften, die dem öffentlichen Ansehen der Sozialwissenschaften nicht wirklich förderlich sein können. 
scheinen, wobei der »Gutmensch « für den gesunden Menschenverstand der Sarrazin-Anhänger bekanntlich der Staatsfeind Nummer Eins ist. ${ }^{10}$

Probleme dieser Art hat die Ökonomie nicht - oder hatte sie nicht bis zum Beginn der Krise. Ausgerüstet mit ihrem Maschinenmodell von Wirtschaft und Gesellschaft, das auf ideale Weise der populären, naturwissenschaftlichen Vorstellung von Wissenschaft entspricht, hat sie bis heute die Kühnheit, mit exakten Vorhersagen der wirtschaftlichen Zukunft bis auf eine Stelle hinter dem Komma aufzuwarten. Nicht nur die "Öffentlichkeit ", sondern auch Staat und Politik waren, wenigstens bis vor kurzem, gerne bereit, ihr zu glauben, dass sie im Besitz einer Technologie der Gesellschaftssteuerung zum Zweck der Wohlstandsvermehrung sei, die der Politik sagen kann, welche Hebel sie bedienen muss, damit hinterher alle besser dastehen. ${ }^{11}$ Dass ausgerechnet die Ökonomen die sozialwissenschaftlichen Gurus unserer Zeit sein würden, hätte man allerdings in den 1970er Jahren nicht erwartet; damals war es eine sich als Planungs- und Gestaltungswissenschaft verstehende Soziologie, die einer »aktiven Gesellschaft « die Instrumente einer »aktiven Politik « zu liefern versprach, mit deren Hilfe sie ihre Zukunft selbst wählen und bestimmen können sollte. In dem Maße jedoch, wie das politische Projekt einer demokratischen Selbststeuerung der modernen Industriegesellschaft scheiterte - später sprach man verschämt von einer »Planungseuphorie ", die damals auch die Wissenden befallen habe -, verlor die Soziologie ihren Status als politische Leitwissenschaft an eine neoklassisch gewendete Wirtschaftswissenschaft, deren Funktion darin bestand, den Übergang vom Sozialdemokratismus zum Neoliberalismus - und damit von politischer zu marktgetriebener Gesellschaftssteuerung - theoretisch zu rechtfertigen. Damit hörte die Soziologie auf, "öffentliche "Soziologie in dem Sinne zu sein, wie sie sich ursprünglich, bei Comte und anderen, selber konzipiert hatte und als welche sie noch von ihren Gründerfiguren der Nachkriegszeit wie Karl Mannheim oder T.H. Marshall betrieben worden war. ${ }^{12}$ Verglichen damit kann die Soziologie von heute nur als privatisiert erscheinen, etwa in ihrer Aufteilung in nach Betroffenengruppen sortierte Subdisziplinen (»Chicano studies «) oder in ihren der Akquise am Forschungsmarkt dienenden »Public Relations «-Anstrengungen mit Hilfe von »nicegood to know «-Wissen, das sich im Wettbewerb um die Konsumenten im Markt für gehobenes Infotainment gegen Evolutionsbiologie und Hirnforschung durchsetzen muss.

Wie kann eine Soziologie, die nicht mehr hoffen kann, Steuerungswissenschaft zu sein, zu einer » öffentlichen « Wissenschaft werden? Welche »Öffentlichkeit « könnte an ihr Interesse haben? Soziologen wissen, dass der öffentliche Raum nicht nur eine Menschenmasse ist, sondern eine soziale und institutionelle Struktur. Gibt es in ihm die aufklärbaren Bürger, die wir uns so gerne als Adressaten von »Gesellschaftsberatung " vorstellen - ein Staats- und Bildungsbürgertum, das willens und in der Lage wäre, an der Erarbeitung einer gesellschaftsumfassenden "öffentlichen Meinung « mitzuwirken? Gibt es noch oder wieder politische Parteien, die ernsthaft daran in-

10 Siehe Lange 2011.

11 Vgl. Streeck 2011 b.

12 Vgl. Mannheim 1951; Marshall 1965 [1949].

Leviathan, 40. Jg., 1/2012 
teressiert sind, etwas über die Welt zu erfahren, und Gewerkschaften, die vorurteilslos nach geprüften Argumenten suchen, die ihre Sache voranbringen könnten? Viel spricht dafür, dass derartige Nachfrager nach Soziologie heute weniger präsent sind als noch vor ein paar Jahrzehnten. Und was ist mit den Medien, durch die hindurch die Soziologie ein öffentliches Interesse an ihr befriedigen müsste, wenn es dies denn gäbe? Das Zeitungssterben in den USA hat dramatische Ausmaße angenommen; hier geht es uns in Europa besser, jedenfalls bisher. Dennoch: Das Fernsehen und neuerdings auch das Internet sind längst an den Printmedien vorbeigezogen. Die »ikonische Wende «, der Vormarsch der Bilder, die noch lange nicht abgeschlossen ist, ist für die Soziologie keine gute Nachricht: Ihre Gegenstände lassen sich nun einmal nicht wirklich fotografieren.

Neurologie und Astronomie sind unvergleichlich besser als die Soziologie, wenn es darum geht, Printredakteure und Fernsehsender mit bunten Bildern zu beliefern. Zudem sind die Medien dabei, sich immer mehr zu spezialisieren, was die »Öffentlichkeit " zunehmend segmentiert. Wenn es in den vergangenen 30 oder 40 Jahren einen Strukturwandel der Öffentlichkeit gab, dann war es einer in Richtung auf eine Multiplizierung von Nischenmärkten. Die heutigen Konsumenten kommerzialisierter Information können sich selbst aussuchen, was sie unterhalten, und ignorieren, was sie langweilen könnte. Die Nutzer der neuesten Medien können sich ihre Nachrichten sogar selbst zusammenstellen oder von einem Algorithmus zusammenstellen lassen, ohne dass irgendjemand mit dem Anspruch eingreifen könnte, besser zu wissen, was ein gewissenhafter Bürger zur Kenntnis nehmen sollte - was bis vor ein paar Jahren die Tageszeitungen und das öffentlich-rechtliche Rundfunkwesen wie selbstverständlich tun konnten und taten. Der heutige Nachrichtenkonsument dagegen erfährt immer mehr nur das, was er erfahren will. Wird sich eine »öffentliche Soziologie " in einer immer mehr fragmentierten Öffentlichkeit nur noch an jene wenden können, die sich, aus welchen idiosynkratischen Gründen auch immer, zufälligerweise für öffentliche Soziologie interessieren?

\section{Soziologie ohne Kapitalismus: Ein Problem des Angebots?}

Dass es Probleme, und zweifellos ernsthafte, auf der Nachfrageseite einer möglichen öffentlichen Soziologie gibt, muss nicht heißen, dass auf der Angebotsseite alles in bester Ordnung wäre - dass also die Soziologie als Sozialwissenschaft tatsächlich Erkenntnisse zu bieten hätte, die man den Bürgern und ihren politischen Vertretern als Pflichtlektüre aufgeben sollte. Ich kann und will nicht beanspruchen, die gesamte Breite unserer Disziplin zu überblicken. Auch neige ich - wie viele andere - dazu, die Themen, über die ich zufällig gerade arbeite, für die wichtigsten zu halten. Dennoch glaube ich sagen zu können, dass die politisch-ökonomische Krise, die seit einigen Jahren die Welt in Atem hält, ein historischer Wendepunkt ist, der unter anderem der Soziologie eine einzigartige Chance bietet, wieder zu einer gesellschaftlich notwendigen Sozialwissenschaft zu werden - vorausgesetzt, sie findet den Weg zurück zu bestimmten fundamentalen Themen und Problemen, die sie im Verlauf 
ihrer historischen Entwicklung anderen Disziplinen, insbesondere der Ökonomie, überlassen hat.

Seit dem Ausbruch der Finanz- und Fiskalkrise des entwickelten Kapitalismus ist den Ökonomen immer wieder, gerade auch von Soziologen, vorgeworfen worden, den Zusammenbruch der Kapitalmärkte und seine Folgen nicht vorhergesehen zu haben. Heute gibt es erstmals seit langem wieder nennenswerte Zweifel in die Fähigkeit der Mainstream-Ökonomie, das Wirtschaftsgeschehen zu erklären und es steuern zu helfen - ausgerechnet jetzt, da sich überall die Erkenntnis zurückmeldet, dass, in den Worten Walther Rathenaus, die Wirtschaft » unser Schicksal « ist. Tatsächlich hat die Weltwirtschaftskrise nicht nur die Ökonomen überrascht, sondern bemerkenswerterweise auch die Soziologen. Während die ersteren von ihren tautologischen Modellen sich selbst stabilisierender freier Märkte in die Irre geführt wurden, hatte die moderne Soziologie die Ökonomie jahrzehntelang fast völlig aus ihrem Blickfeld verbannt und sie, nach Maßgabe des von Talcott Parsons in den 1950er Jahren in ihrem Namen abgeschlossenen interdisziplinären Waffenstillstands, ${ }^{13}$ der Ökonomie überlassen. Wenn die Krise von 2008 ein neues Bewusstsein von der Wichtigkeit der Wirtschaft für die moderne Gesellschaft geschaffen hat, dann war die moderne Soziologie in einer denkbar schlechten Ausgangsposition, auf dieses zu reagieren, nachdem sie jahrelang ihren Forschungsgegenstand als ökonomiefreie Gesellschaft definiert hatte. Dabei wurden wichtige disziplinäre Traditionen marginalisiert oder gänzlich ausgelagert, wie zum Beispiel die politische Ökonomie, die der effizienztheoretischen Ökonomie überlassen wurde, deren Hegemonie in diesem Bereich heute nur noch von institutionalistisch orientierten Politikwissenschaftlern infrage gestellt wird. ${ }^{14}$ Jüngere Versuche, die Ökonomie auf dem Weg über die Etablierung einer »Wirtschaftssoziologie « als neuer Subdisziplin zurückzuholen, beschränkten sich allzu lange darauf, alternative Rezepte zur Steigerung der Effizienz wirtschaftlicher Transaktionen zu entwickeln, etwa in Gestalt einer Ergänzung von Märkten durch » Netzwerke «. ${ }^{15}$

Ich sehe in der Krise ein starkes Signal dafür, dass ein soziologisches Forschungsprogramm, das eine im Wesentlichen ökonomiefreie Gesellschaft als Gegenstand hat, unhaltbar geworden ist - es sei denn, wir wollten weiterhin gegenüber den

\section{Camic 1991.}

14 Siehe etwa Thelen 2002; 2004.

15 Granovetter 1985; zur Kritik der »neuen Wirtschaftssoziologie« siehe Beckert 2003, 2009; Beckert, Streeck 2008. Nicht zuletzt zeichnet sich vor allem die in den Vereinigten Staaten gepflegte Version der "new economic sociology « durch eine bemerkenswerte Vernachlässigung des Politischen oder auch Staatlichen in der Wirtschaft aus, eine Tendenz, die sie mit großen Teilen der neueren Soziologie insgesamt teilt. Ebenso wie gegen die Entökonomisierung der Soziologie könnte man auch gegen ihre Entpolitisierung polemisieren - bzw. gegen eine beklagenswerte Tendenz der akademischen Soziologie in der zweiten Hälfte des 20. Jahrhunderts, sowohl das politische als auch das ökonomische "Subsystem " der Gesellschaft an andere, jeweils auf diese spezialisierte sozialwissenschaftliche Disziplinen abzutreten, das eine an die Politikwissenschaft, das andere an die Wirtschaftswissenschaft. Auf der Strecke blieb dabei das Projekt einer politischen Ökonomie als Gesellschaftstheorie. 
wichtigsten gesellschaftlichen Entwicklungen unserer Zeit so sprachlos bleiben wie vor den Ereignissen von 2008. ${ }^{16}$ Viele haben heute das Gefühl, dass die finanzielle und fiskalische Krise nicht nur eine ökonomische, sondern auch und primär eine gesellschaftliche Angelegenheit ist, und zwar eine, die wichtig genug ist, um nach einer revidierten Interpretation der modernen Gesellschaft zu verlangen. $\mathrm{Zu}$ den Phänomenen, denen diese Rechnung zu tragen hätte, gehören die fortwährende Revolutionierung des Gesellschaftlichen durch expandierende Märkte, die sich aus ihr ergebende Fragilität sozialer Strukturen und politischer Institutionen, die wachsende Unsicherheit, der Regierungen und Bürger ausgesetzt sind, weil sich die mittlerweile global integrierten Märkte immer mehr der sozialen Kontrolle entziehen, und die Ungeeignetheit des Marktes als Ort sozialer Integration und als Grundlage gesellschaftlicher Ordnung. ${ }^{17}$

Grundsätzlich sollte die Soziologie - mit ihrer Tradition als kritische Theorie der Moderne - diesem Anspruch gerecht werden und der »Öffentlichkeit « Erkenntnisse anbieten können, die diese nur zu ihrem Nachteil ignorieren könnte. Dazu allerdings müsste sie, so meine These, die Ökonomie als zentralen Gegenstand einer jeden Gesellschaftstheorie rehabilitieren, die diesen Namen verdient - und in ihr nicht nur einen neutralen, von esoterischen Naturgesetzen regierten und von wissenschaftlich informierten Technokraten steuerbaren Mechanismus der Wohlstandsmehrung sehen. Das wird ihr aber nicht gelingen, ohne dass sie ihren Waffenstillstand mit der Ökonomie aufkündigt und jene politische Ökonomie wiederentdeckt, die die Soziologie war, als sie noch jung war, und die sie später aufgegeben hatte, um sich auf die »Gesellschaft « zu spezialisieren. Einen besseren Zeitpunkt als den jetzigen werden wir dafür nicht finden.

Warum hat die Soziologie die Ökonomie überhaupt den Ökonomen überlassen? Wie konnten wir glauben, dass eine ökonomiefreie Gesellschaft ein lohnender Forschungsgegenstand sein könnte? Es ist interessant, dass die Verbannung der Ökonomie aus der Soziologie in Deutschland früher stattgefunden hat als in den USA, und zwar nicht aus wissenschaftlichen, sondern zu einem guten Teil auch aus politischen Gründen. Max Weber hatte einen Lehrstuhl für Nationalökonomie und war Mitglied des Vereins für Socialpolitik, der professionellen Vereinigung der Ökonomen. Dass er später aus dieser austrat und die Deutsche Gesellschaft für Soziologie (DGS) gründete, war politisch motiviert: Weber war ein Gegner der von den Ökonomen im Verein für Socialpolitik öffentlich proklamierten reformistischen Sozialpolitik. Die DGS sollte ihn davor bewahren, mit den ihm verhassten Kathedersozialisten umgehen zu müssen, weswegen sich die DGS satzungsmäßig zur »Wert-

16 Ausnahmen bestätigen die Regel. Für die Zeit nach 2008 siehe unter anderem Krippner 2011; Calhoun, Derluguian 2011.

17 Siehe Streeck 2009. 
freiheit " verpflichten musste. ${ }^{18}$ In der Praxis erwies sich dies jedoch als nicht durchsetzbar. Als die "soziale Frage « bei den Tagungen der DGS immer wieder hochkam, verabschiedete sich Weber, der vergeblich versucht hatte, das Thema zu unterdrücken, aus der organisierten Soziologie. ${ }^{19}$ Wenige Jahre später starb er.

Bemerkenswerterweise hat die deutsche Soziologie nach Webers Tod die großen Themen von Wirtschaft und Gesellschaft, die wir heute mit seinem Namen verbinden, nicht mehr aufgegriffen. Man überließ sie weitgehend der institutionalistischen Wirtschaftstheorie der Historischen Schule, etwa Werner Sombart, der in der Weimarer Soziologie im engeren Sinne nur am Rande eine Rolle spielte, wenn überhaupt. Der Niedergang der Historischen Schule nach der nationalsozialistischen Machtergreifung und nicht zuletzt ihre versuchte Annäherung an den Nationalsozialismus ermöglichten nach dem Krieg den Aufstieg der »theoretischen « - im Gegensatz zur historischen - Wirtschaftswissenschaft. Was die Soziologen angeht, die in den 1920er Jahren damit beschäftigt waren, sich an den deutschen Universitäten zu etablieren, so achteten diese darauf, nicht für Sozialisten gehalten zu werden oder für Marxisten, was damals mehr oder weniger dasselbe war. Max Horkheimer und seine Mitarbeiter am Institut für Sozialforschung in Frankfurt sahen sich nicht als Soziologen und scheinen nie erwogen zu haben, der DGS beizutreten. Die theoretische Soziologie der Weimarer Republik, soweit sie sich durch die Soziologentage der Öffentlichkeit bekanntmachte, bestand überwiegend aus einer formalistischen Theorie sozialer Beziehungen (Leopold von Wieses »Beziehungslehre«), von der heute nichts mehr übriggeblieben ist. Die empirische Soziologie beschäftigte sich hauptsächlich mit demografischer Forschung und, als sogenannte Siedlungsforschung, insbesondere mit Siedlungsstrukturen in Deutschland und zunehmend in Mittel- und Osteuropa. Anders als im Nachkriegsmythos der Disziplin behauptet, ging es der empirischen Soziologie im Dritten Reich alles andere als schlecht; sie genoss den Respekt von Staat und Partei, vor allem wenn sie sich mit Stadt- und Landplanung in den annektierten Gebieten im Osten beschäftigte. Kapitalismus

18 Webers eigene Sozialwissenschaft war bekanntlich alles andere als » wertfrei «. Seine leidenschaftliche Ablehnung der Befürwortung der Sozialpolitik durch die zeitgenössische Volkswirtschaftslehre war die eines liberalen Nationalisten, für den die von ihm erwarteten Konflikte vor allem mit Großbritannien um das nationale Überleben und um internationale Vormacht von überragender Bedeutung waren (Weber 1895). Sozialpolitik und Demokratie waren für Weber nicht dazu da, die Menschen glücklich zu machen, sondern sollten helfen, das neugegründete Deutsche Reich für eine anarchische und kriegerische internationale Welt zu rüsten. Dass Weber seine Position für wertfrei halten konnte, lag daran, dass er »Realpolitik « für eine objektive Tatsache hielt und nicht für etwas, für oder gegen das man sich entscheiden könnte.

19 Vgl. Fritz, Mikl-Horke 2007.

Leviathan, 40. Jg., 1/2012 
spielte in dieser speziellen Form einer »öffentlichen Soziologie« natürlich keine Rolle. ${ }^{20}$

Nach dem Zweiten Weltkrieg hielt sich die Soziologie weiter von der Ökonomie fern, ungeachtet dessen, dass diese radikal mit der Tradition der Historischen Schule brach und immer »modellplatonistischer « wurde. ${ }^{21}$ Die institutionalistische Ökonomie verschwand, bis sie in jüngerer Zeit unter "modernen ", effizienztheoretischen Vorzeichen zurückkehrte. Historische wirtschaftswissenschaftliche Forschung wurde marginalisiert, selbst in ihrer späteren Gestalt als historische Ökonometrie, auch "Kliometrie " genannt. Was Soziologie war und was nicht, wurde zunehmend in den USA entschieden, von wo die Disziplin reimportiert wurde, nachdem die Weimarer und die nationalsozialistische Soziologengeneration verstorben oder in den Ruhestand gegangen war. In den 1960er Jahren, als das Nachkriegswachstum seinen Höhepunkt erreichte, schien die Wirtschaft nicht mehr von soziologischem oder auch politischem Interesse zu sein. Viele glaubten, wie von Keynes in seinen optimistischeren Momenten erhofft, dass die Wirtschaftswissenschaft zu so etwas wie Zahnmedizin geworden war: eine Profession, die man zu Rate zieht, wenn man ein Problem hat, im Besitz erprobter Techniken, mit denen sich schmerzfrei reparieren lässt, was repariert werden muss. ${ }^{22}$

In Frankfurt, wo ich 1966 zu studieren begann, war der Kapitalismus denn auch von Adorno in » Tauschgesellschaft « umbenannt worden, und bis auf ein paar orthodox-kommunistische Außenseiter unter den Studenten glaubte niemand, dass »das System « jemals wieder für ökonomische Krisen anfällig werden könnte (die Krise der Saison war bekanntlich die Legitimitätskrise ${ }^{23}$ ). Der Glaube, dass die Ökonomie im Wesentlichen nur noch eine technische Frage war, war allerdings keineswegs auf Frankfurt beschränkt, sondern wurde weithin geteilt, von Soziolo-

20 Mit etwas Pech hätte die Soziologie zu einer tragenden Säule des nationalsozialistischen Regimes werden können. 1934 trat die DGS erstmals seit der Machtergreifung der Nazis zusammen. Die Frage war, ob man unter einer neuen, pro-nationalsozialistischen Führung weitermachen oder die Vereinigung aus Protest auflösen sollte. Eine Reihe von Anwesenden sprach sich dafür aus, einen gewissen Reinhard Höhn zum Nachfolger des damaligen DGS-Präsidenten Ferdinand Tönnies zu wählen. Höhn, ein Jurist, der als Assistent des Soziologen Franz Wilhelm Jerusalem in Jena arbeitete, wurde später zum Professor für Staatsrecht ernannt und zu einer führenden Figur im SS-Hauptquartier, wo er einer Abteilung des Reichssicherheitshauptamts vorstand. Höhn erhielt nicht genug Stimmen, doch um sich die neue Regierung nicht zum Feind zu machen, sahen die Mitglieder davon ab, die DGS aufzulösen, und beschlossen stattdessen, die Aktivitäten der Vereinigung zeitweilig einzustellen. Erst nach 1945 wurde die DGS unter Führung von Wieses wiederbelebt. Höhn tauchte in den 1950er Jahren wieder auf, als er die Akademie für Führungskräfte der Wirtschaft in Bad Harzburg gründete, eine der führenden Management-Schulen der Bundesrepublik, die er bis weit in die 1970er Jahre hinein leitete. Siehe neuerdings van Dyk, Schauer 2010; Schauer, van Dyk 2010.

21 Vgl. Albert 1965.

$22 »$ Economics ... should be a matter for specialists, like dentistry. If economists could manage to get themselves thought of as humble, competent people, on a level with dentists, that would be splendid! (Keynes 1963 [1930], S. 373).

23 Vgl. Habermas 1973; Offe 1972. 
gen nicht weniger als von Ökonomen. Ein Beispiel unter vielen ist Amitai Etzionis Buch The Active Society aus dem Jahr 1968 - der wohl ehrgeizigste Versuch, die Bedingungen zu benennen, unter denen moderne demokratische Gesellschaften die Richtung ihrer Entwicklung selber bestimmen können. Auf seinen 666 Seiten erwähnt das Buch die Ökonomie nur ein einziges Mal, und auch nur um festzustellen, dass »die westlichen Nationen « heute auf ihre Fähigkeit vertrauen können, "gesamtgesellschaftliche Prozesse durch umfassenden Einsatz keynesianischer und anderer Instrumente zur Verhinderung von unkontrollierter Inflation und von Depressionen und zur Erzeugung von Wirtschaftswachstum zu steuern «. ${ }^{24}$

Wie gesagt, ich bin überzeugt, dass die splendid isolation der Soziologie gegenüber der Ökonomie nicht mehr durchzuhalten ist, es sei denn, sie wollte sich damit abfinden, gegenüber den großen Themen der Zeit in Bedeutungslosigkeit zu versinken. Angesichts der Krise sollte sie zugeben, dass ihre Wette auf das Nicht-Ökonomische in der Gesellschaft nicht aufgegangen ist. Da die Soziologie, anders als die Standardökonomie, noch nicht völlig auf »Rational Choice « hereingefallen ist, kann sie sich leichter als diese von einem Weltbild lösen, in dem die rationale Verfolgung individueller Interessen fähig sein soll, eine stabile Ordnung hervorzubringen. Ebenso wenig sind Soziologen auf funktionalistische Gleichgewichtsmodelle der Gesellschaft angewiesen, sollten also in der Lage sein, die inhärente Rastlosigkeit und Krisenanfälligkeit der modernen Wirtschaftsgesellschaft - in anderen Worten: des heutigen Kapitalismus - zu verstehen. Vor allem aber können wir auf ältere Konzepte des Kapitalismus zurückgreifen, in denen dieser als historische soziale Formation verstanden wird, als real existierende, sich dynamisch entwickelnde soziale und institutionelle Struktur ${ }^{25}$ statt als idealtypisches universalistisches Wirtschaftsmodell oder als Synonym für Marktwirtschaft, wie in der Wirtschaftstheorie oder in der ökonomistischen Abteilung der "Spielarten-des-Kapitalismus «-Literatur ${ }^{26}$. Man muss sich nur daran erinnern, dass noch in den 1970er Jahren ein Daniel Bell Traditionen soziologischer Kapitalismustheorien wachhielt und weiterzuentwickeln imstande war, die auf Marx, Weber, Sombart, Schumpeter und andere zurückging, ${ }^{27}$ mit gelegentlichen Grüßen über die ideologischen Schützengräben hinweg an den einen oder anderen Neomarxisten wie etwa James O'Connor. ${ }^{28}$

\section{Öffentliche Soziologie als Rückkehr zur politischen Ökonomie}

Was also hätte eine Soziologie, die sich anschickte, ihre Tradition als politische Ökonomie wiederzubeleben, einer zeitgenössischen Öffentlichkeit zu sagen - einer Öffentlichkeit, die sich mehr denn je zu fragen begonnen hat, in welche Richtung sich der moderne Kapitalismus entwickelt? Zum Mindesten sollten wir in der Lage

\section{Etzioni 1968, S. 10.}

25 Siehe Streeck 2011 c.

26 Siehe Hall, Soskice 2001.

27 Siehe Bell 1976.

28 Siehe O’Connor 1973.

Leviathan, 40. Jg., 1/2012 
sein, dem öffentlichen Bewusstsein einzuprägen, dass die derzeitige Krise kein Zufall ist - nicht das bedauerliche Resultat von versehentlichem Missmanagement auf dem amerikanischen Immobilienmarkt -, sondern aus fundamentalen Spannungen und Widersprüchen innerhalb des Regimes des demokratischen Kapitalismus erwächst, wie wir es in den westlichen Gesellschaften seit dem Ende des Zweiten Weltkriegs gekannt haben..$^{29}$ Die Inflation in den Siebzigern, die steigende Staatsverschuldung in den Achtzigern, die Deregulierung des privaten Kreditwesens in den Neunzigern als Kompensation für eine erste Welle fiskalischer Konsolidierungsanstrengungen und die heutigen Versuche, unter dem Druck einer globalisierten haute finance die öffentlichen Haushalte für ihre Kreditgeber vertrauenswürdig zu machen - all das ist Ausdruck des Zusammenpralls einer lebensweltlichen moralischen Ökonomie der Sozial- und Bürgerrechte mit einer kapitalistischen ökonomischen Ökonomie, die nach Marktgerechtigkeit verlangt. Der Schauplatz dieses Konflikts hat sich über die Jahrzehnte immer wieder verlagert, von den Arbeitsbeziehungen und Tarifverhandlungen auf dem Arbeitsmarkt zur Partei- und Wahlpolitik hin zu den Märkten für Konsumentenkredite und schließlich den internationalen Finanzmärkten und den gegenwärtigen Auseinandersetzungen um die Bedienung und Refinanzierung der nach 2008 sprunghaft angestiegenen Staatsschulden. Obwohl das Problem immer dasselbe war - in der Terminologie David Lockwoods, wie mit den widersprüchlichen Anforderungen von Systemintegration und sozialer Integration in einer kapitalistischen Gesellschaft umzugehen sei $-{ }^{30}$ kann nicht übersehen werden, dass die marktkorrigierende Kraft der Demokratie und ihrer Organisationen, vor allem der Gewerkschaften und politischen Parteien, mit jeder Krise kontinuierlich abgenommen hat. Heute werden die Widersprüche des demokratischen Kapitalismus in der Arena der internationalen Finanzdiplomatie, also zwischen Staaten und Investmentbanken verhandelt - einem Milieu, das gegen den Druck der Öffentlichkeit und den strategischen Zugriff des Mannes bzw. der Frau »auf der Straße" so gut wie immun und dessen Logik für Außenstehende, von einigen Spezialisten abgesehen, völlig undurchschaubar ist.

Von Soziologen wird man nicht notwendig erwarten, dass sie wissen, wie das Geldwesen neu geordnet und das Wirtschaftswachstum wiederhergestellt werden kann. Doch sie können uns helfen zu verstehen, dass viel mehr auf dem Spiel steht als das und dass die Wiederherstellung des Sozialpakts des demokratischen Kapitalismus, von dem die Legitimität unserer sozialen Ordnung abhängt, die Fähigkeiten jeder noch so sachkundigen Wirtschaftspolitik übersteigt. Die Erwartungen der Investoren sind nicht die einzigen, die im Spiel sind, und ganz sicher nicht die einzig legitimen. Im Gegensatz zu den meisten Ökonomen können Soziologen wissen, dass die Aufgaben der Politik komplexer sind, als einer widerstrebenden Gesellschaft Marktgerechtigkeit nach Maßgabe von Grenzproduktivität und relativen Preisen aufzuzwingen. Politik - oder doch demokratische Politik - muss zwischen den Bedürfnissen der menschlichen Lebenswelt und denen des "Kapitals « einen Ausgleich finden. Wenn das Vertrauen der Investoren auf Kosten des Vertrauens der Bürger

29 Zum Folgenden siehe Streeck 2011 a.

30 Lockwood 1964. 
gewonnen wird, dann ist für die soziale Stabilität am Ende nichts erreicht. Während heute die politischen und ökonomischen Eliten versucht sein mögen, die Krise zu nutzen, um den Kapitalismus ein für alle Mal gegen die Demokratie zu immunisieren, verfügt die Soziologie über das begriffliche Handwerkszeug, um die Öffentlichkeit über die Risiken einer solchen Strategie aufzuklären. Sie sollte sich verpflichtet fühlen, davon Gebrauch zu machen.

Inzwischen zeichnet sich immer deutlicher $a b$, dass wir es nicht nur mit einer schweren Wirtschaftskrise zu tun haben, sondern auch mit einer Demokratiekrise. In Europa übertragen nationale Regierungen unter dem Druck der Finanzmärkte ihre Entscheidungsgewalt auf internationale Organisationen und entmachten damit die nationalen Parlamente und, in letzter Konsequenz, die Wähler. Schuldnerstaaten haben keine Alternative, als das Diktat ihrer Gläubiger zu akzeptieren; Wahlen verlieren in diesen Ländern ihren Sinn. ${ }^{31}$ Die Gläubigerstaaten wiederum werden von »den Märkten " getrieben; sie müssen schnell und flexibel auf deren wechselnde Forderungen reagieren, was ihren Parlamenten immer weniger Zeit lässt, ihre demokratischen Rechte auszuüben. Die international institutionalisierte Austeritätspolitik verringert drastisch den politischen Spielraum und macht politische Partizipation zunehmend folgenlos. Bemerkenswert in diesem Zusammenhang ist, dass schon die Wahlbeteiligung seit den 1990er Jahren in nahezu allen europäischen Ländern und auf allen politischen Ebenen kontinuierlich gesunken ist, von den Kommunen bis zum sogenannten Europäischen Parlament, und am stärksten in Regionen mit hoher Armut, vielen Einwanderern und zerrütteten Familien, also dort, wo politische Mobilisierung eigentlich am nötigsten wäre. ${ }^{32}$

Heute ist es fast schon ein Gemeinplatz, dass die aktuelle Krise in einem erheblichen Ausmaß eine Vertrauenskrise ist - in Bezug auf den Wert des Geldes, die Bereitschaft und Fähigkeit von Schuldnern, ihre Schulden zurückzuzahlen, die Fähigkeit der politischen Führungen, dem Druck »der Märkte« standzuhalten, und die der Märkte, für eine effiziente, um nicht zu sagen faire Ressourcenallokation zu sorgen. Nicht nur, dass die Zuversicht gering ist, dass unsere Regierungen und internationalen Organisationen in der Lage sind, weitere Krisen zu verhindern. Hinzu kommt ein rapider Verlust an Vertrauen zwischen den Marktakteuren selber, vor allem den Banken, deren Geschäft es notwendig macht, dass sie einander Geld leihen. Ergebnis könnte sein, dass Staaten und Zentralbanken ein weiteres Mal als Kreditgeber letzter Instanz einspringen und faule Kredite übernehmen und Garantien in einem Ausmaß abgeben müssen, das ihnen endgültig zum Verhängnis werden könnte. Es ist noch nicht lange her, dass die Transaktionskosten-Ökonomik mit der Behauptung reüssierte, dass Institutionen am besten von den an effizienten Austauschbeziehungen interessierten Marktakteuren selbst, also »von unten « geschaf-

31 Ein paar Monate nach der Konferenz, auf der dieser Vortrag gehalten wurde, wurden die Regierungschefs zweier "Schuldnerländer « der Eurozone auf Druck der Kapitalmärkte und der Europäischen Union durch Finanzmanager ersetzt. Einer der beiden Geschassten hatte vorher ein Referendum über die von seinem Land verlangte Austeritätspolitik abhalten wollen, musste dieses Vorhaben dann aber aufgeben.

32 Siehe Schäfer 2010.

Leviathan, 40. Jg., 1/2012 
fen werden. Der Rational-Choice-Institutionalismus in den Wirtschafts- und Politikwissenschaften hat diese Botschaft bereitwillig verinnerlicht, indem er staatliche Regierung durch private »Governance " ersetzte. ${ }^{33}$ Die Krise hat gezeigt, dass private Selbstorganisation als Mittel zur Herstellung sozialer Ordnung sehr schnell an ihre Grenzen stößt. Wenn sie zusammenbricht, ist zur Durchführung der nötigen Reparaturarbeiten wieder die öffentliche Gewalt gefragt. Es gibt für die Soziologie keinen Grund, nicht immer wieder auf den offenkundigen Bankrott der liberalen Institutionen-Theorien und die sich aus ihm ergebende Bestätigung des Durkheim'schen soziologischen Erbes hinzuweisen.

Karl Polanyi, dessen Werk eine wachsende Zahl von Soziologen inspiriert, war in den 1950er und 1960er Jahren nicht daran interessiert, als Soziologe zu gelten und der soziologischen Profession anzugehören. Ihm reichte es, Ökonom, Wirtschaftshistoriker und Sozialanthropologe zu sein. Es sagt etwas aus über unsere Disziplin, dass sie ihn erst in den 1990er Jahren entdeckt hat, auf dem Höhepunkt des Neoliberalismus und zu einer Zeit, als die globale Finanzialisierung eine weitere revolutionäre Transformation der kapitalistischen Wirtschaft in Gang gesetzt hatte. Tatsächlich gibt es für unsere heutigen Probleme keine bessere zusammenfassende Darstellung als eine, die sich an Polanyis Konzept der drei »fiktiven Waren « - Geld, Natur und Arbeit - und die Grenzen ihrer Kommodifizierung anlehnt. ${ }^{34}$ Viele haben sich davon überzeugt, dass diese Grenzen gegenwärtig bereits erreicht sind, und mit ihnen die weiteren kapitalistischen Wachstums, zumindest eines Wachstums, das noch halbwegs mit menschlichen Bedürfnissen vereinbar wäre. So hat die privatwirtschaftliche Produktion von Geld im Zuge der Deregulierung der Finanzindustrie ganze Gesellschaften beispielloser Unsicherheit unterworfen, Verteilungskonflikte in und zwischen ihnen verschärft und bislang ungelöste Probleme einer globalen Re-Regulierung aufgeworfen. Was die Natur angeht, so haben wir allmählich begriffen, dass auch auf sie das grundlegende Merkmal einer fiktiven Ware zutrifft, nämlich dass ihr Angebot der Nachfrage nach ihr nicht beliebig folgen kann. Alles spricht dafür, dass wir Wege finden müssen, unsere globalen öffentlichen Güter vor weiterer Kommodifizierung zu schützen, wenn wir verhindern wollen, dass unsere Lebensgrundlagen im Dienste ungezügelter Kapitalakkumulierung aufgefressen werden. Und schließlich hat die immer weiter fortschreitende Flexibilisierung der Märkte für Arbeit und der Arbeitsorganisation Individuen und Familien einem unerbittlichen Druck unterworfen, ihre Lebensweise nach Maßgabe immer unberechenbarerer Anforderungen immer kompetitiverer Märkte einzurichten. Das Ergebnis ist unter anderem eine zunehmende Abkoppelung einer verarmten »Überschuss «-Bevölkerung sozialer Verlierer vom gesellschaftlichen Leben; überarbeitete Mittelschicht-Familien, die trotz eines in der Geschichte beispiellosen Wohlstands immer mehr und immer intensivere Arbeitsstunden ableisten; und eine kleine Elite von »winner-take-all «-Superreichen, deren Habgier keine Grenzen kennt und deren Boni und Dividenden längst aufgehört haben, der Gesellschaft irgendeinen Nutzen zu bringen.

33 Vgl. Offe 2008.

34 Vgl. Polanyi 1957 [1944], S. 68-77. 
Was, wenn nicht eine politische Ökonomie des Kapitalismus, wie sie von Polanyi in seinem Konzept der Grenzen der Kommodifizierung vorweggenommen wurde, sollte das Thema einer erneuerten »öffentlichen Soziologie « sein? Natürlich wartet auf uns eine Menge Arbeit. Die Soziologie hat zum Thema Geld und Finanzen gerade in den Jahrzehnten der »Finanzialisierung « nur wenig beigetragen - abgesehen von ein paar unterhaltsamen, aber politisch unergiebigen ethnografischen Beschreibungen des Arbeitslebens von Händlern an der Wall Street, verfasst vor dem Ausbruch der Krise. ${ }^{35}$ In Bezug auf Fragen der natürlichen Umwelt haben Soziologen Unmengen von Studien darüber verfasst, warum und wann Menschen gewillt sind, Müll zu trennen oder andere wenig kostspielige Beiträge zum Umweltschutz zu leisten. Aber die Frage, was es ist, das unsere Gesellschaften so abhängig von kapitalistischem Wachstum macht, selbst um den Preis der Zerstörung ihrer ökonomischen, ökologischen und menschlichen Grundlagen, haben wir seltsamerweise den wenigen heterodoxen Ökonomen überlassen. Ähnliches gilt für das Nachdenken darüber, wie eine Gesellschaft, die zum Wachstum gezwungen scheint, zu einer werden könnte, die im Einklang mit der Natur und sich selbst lebt. ${ }^{36}$ Dass Ökonomen, wenn sie gelegentlich nach nichtmaterialistischen Quellen menschlicher Zufriedenheit fragen, sich an die Psychologie wenden, kann man ihnen nicht vorwerfen angesichts der Tatsache, dass die Soziologie das Thema bislang gründlich gemieden hat - obwohl man durchaus darauf bestehen könnte, dass es sich dabei mindestens ebenso sehr um ein soziales und politisches wie um ein psychologisches Thema handelt. Vergleichsweise gut steht die Soziologie nur beim Thema Arbeitsmärkte und Familienstrukturen da und bei der Frage der Konflikte zwischen der Teilnahme an einem immer härteren Konkurrenzkampf um Einkommen und gehobenen Konsum auf der einen Seite und den Anforderungen des sozialen Lebens, einschließlich der Kindererziehung, auf der anderen. ${ }^{37}$

\section{Noch einmal die Nachfrageseite}

Das bringt mich zu meiner Ausgangsfrage zurück: Würde überhaupt jemand zuhören? Natürlich sollten wir nicht allzu optimistisch sein. Doch dies sind keine normalen Zeiten, und vielleicht sind die normalen Zeiten auch vorerst ohnehin vorbei. Unter Eliten wie unter Bürgern - und sogar in der Wissenschaft - hat sich ein Gefühl der Krise ausgebreitet, das über das hinausgeht, was wir aus den letzten Jahrzehnten kennen. Vielleicht nähern wir uns tatsächlich, historisch-institutionalistisch gesprochen, einer critical juncture: einer Periode beschleunigten Wandels mit ungewissem Ausgang, die für lange Zeit von prägender Bedeutung sein wird. Ein Symptom hierfür könnte sein, wie ratlos die ökonomische Standardtheorie ist, wenn es darum geht, wie dem seit 2008 anhaltenden globalen wirtschaftlichen Desaster zu begegnen sei. Nie waren die führenden Ökonomen der Welt so uneins wie heute - etwas, das

35 Etwa Abolafia 1997, siehe aber unter anderen Ingham 2004.

36 Siehe - außerhalb des Mainstreams - Miegel 2010.

37 Für viele andere siehe Hochschild 1997, 2003.

Leviathan, 40. Jg., 1/2012 
selbst ihre publizistischen Zentralorgane, allen voran der Economist und die Financial Times, zur Kenntnis nehmen müssen. Möglicherweise ist die Erklärung, dass die Trickkiste kapitalistischer Wirtschaftspolitik in der Demokratie nunmehr leer ist und die Wirtschaftstheorie, wie wir sie kennen, deshalb ihre Herrschaft über den öffentlichen Diskurs zu verlieren beginnt. Auch innerhalb der Ökonomie als Disziplin scheinen sich Zweifel auszubreiten, etwa was ihre Art der Wohlstands- und Wachstumsmessung angeht oder allgemein die Aussichten auf einen stetig wachsenden materiellen Wohlstand. ${ }^{38}$

Die Ökonomie wieder in die Gesellschaft und in die Soziologie zurückzuholen könnte ein Programm sein, für das wir Verbündete finden könnten. Vielleicht findet es sogar bei den politischen Eliten Anklang, in einer Welt, in der die Staaten dabei sind, in so etwas wie Aktiengesellschaften verwandelt zu werden, die das Vertrauen ihrer Kapitalgeber verdienen müssen; in der internationale Organisationen als Anlagensicherungssysteme oder Inkassobüros für private Investoren auftreten; und in der Regierungen sich wie Unternehmensvorstände vorkommen müssen, die ihren zu disziplinierten Schuldenzahlern umerzogenen Bürgern creditor value abzupressen haben. Vielleicht könnte sich sogar unter den Jüngeren, die nicht mehr in Parteien und Gewerkschaften eintreten und aufgehört haben, zur Wahl zu gehen, ein Bedürfnis nach einer neuen kritischen Theorie der politischen Ökonomie entwickeln. Wie bei jeder "Grundlagenforschung " gilt auch hier, dass der Umstand, dass wir nicht wissen, wer sie nutzen wird, kein Grund sein kann, mit ihr nicht anzufangen.

Damit Soziologie wirklich öffentliche Soziologie werden kann, muss sie sich für den Moment bereithalten, in dem die Grundlagen der modernen Wirtschaftsgesellschaft neu gedacht werden müssen, wie im New Deal oder in den Jahren unmittelbar nach dem Zweiten Weltkrieg. Ein solcher Moment könnte in der Tat bevorstehen. Selbst wenn unser einziges Publikum zunächst die akademische Welt wäre, heißt das nicht, dass unsere Bemühungen vergeblich sein müssen. Im letzten Kapitel seiner General Theory spricht Keynes von der Macht der »Gedanken der Ökonomen und Staatsphilosophen, sowohl wenn sie im Recht, als auch wenn sie im Unrecht sind «. Die Welt, so Keynes, » wird in der Tat durch nicht viel anderes regiert ", selbst wenn neue Ideen nicht immer sofort wirksam werden: »Im Bereich der Wirtschaftslehre und der Staatsphilosophie gibt es nicht viele, die nach ihrem fünfundzwanzigsten oder dreißigsten Jahr durch neue Theorien beeinflußt werden, so daß die Ideen, die Staatsbeamte und Politiker und selbst Agitatoren auf die laufenden Ereignisse anwenden, wahrscheinlich nicht die neuesten sind. Aber früher oder später sind es Ideen, und nicht eigennützige (Gruppen-)Interessen, von denen die Gefahr kommt, sei es zum Guten oder zum Bösen «.39

Wir können nicht wissen, und vielleicht gibt es gute Gründe, zu bezweifeln, ob das Keynes'sche »trickle-down «-Modell ideellen Wandels genügend Zeit haben wird, um uns noch zu Hilfe zu kommen: jenes »allmähliche Sich-Aufdrängen « von neuen Ideen, das für Keynes selber so gut funktioniert hat. Aber das kann nur bedeuten, dass wir nicht früh genug damit anfangen können, die intellektuelle Vor-

38 Vgl. Stiglitz et al. 2009.

39 Keynes 2009 [1936], Kap. 24. 
herrschaft der ökonomischen Standardtheorie über das zeitgenössische Verständnis von Wirtschaft und Gesellschaft infrage zu stellen. Die erste Öffentlichkeit einer als politische Ökonomie erneuerten »öffentlichen Soziologie « wird zweifellos die akademische Welt sein, mit ihrer bislang nie gekannten Zahl von Studenten der Volksund Betriebswirtschaftslehre, denen heute im Wesentlichen beigebracht wird, Gesellschaft als Gelegenheit zur Nutzenmaximierung für diejenigen zu sehen, die die rationalsten - im Sinne von egoistischsten - Entscheidungen zu treffen vermögen. Wenn wir hier keine Zweifel sähen können, wo dann? Der Parsons'sche Waffenstillstand zwischen Soziologie und Ökonomie hat den Kant'schen Streit der Fakultäten an einer Stelle zum Schweigen gebracht, wo wir ihn heute dringender denn je brauchen. Eine wachsende Zahl von Soziologen und Politikwissenschaftlern ${ }^{40}$ hat in den letzten Jahren begonnen, gemeinsam mit heterodoxen Ökonomen verschiedenster Couleur an einer neuen politischen Ökonomie zu arbeiten, einer Sozioökonomie, in der das Ökonomische dem Sozialen untergeordnet ist und nicht umgekehrt, zunächst als theoretisches und dann, hoffentlich, auch als politisches Projekt. Höchste Zeit für die Soziologie als Disziplin, sich auf ihre Anfänge rückzubesinnen, auch und gerade in Zeiten einer kapitalistischen Neuordnung der Universitäten vor allem in Großbritannien, aber nicht nur dort -, deren Ziel die Eliminierung kritischer Reflexion ist, natürlich für keinen anderen Zweck als den der Steigerung der ökonomischen Effizienz. Wenn sich eine erneuerte »öffentliche Soziologie" selbst in der Öffentlichkeit der Akademie kein Gehör verschaffen könnte, wie kann sie dann hoffen, in der Welt von Youtube, Facebook, Fox TV und Bild-Zeitung bestehen zu können?

\section{Literatur}

Abolafia, Mitchel Y. 1997. Making markets: opportunism and restraint on Wall Street. Cambridge: Harvard University Press.

Albert, Hans 1965. "Modell-Platonismus. Der neoklassische Stil des ökonomischen Denkens in kritischer Beleuchtung ", in Logik der Sozialwissenschaften, hrsg. v. Topitsch, Ernst, S. 406-434. Köln, Berlin: Kiepenheuer \& Witsch.

Beckert, Jens 2003. "Economic sociology and embeddedness. How shall we conceptualize economic action? «, in Journal of Economic Issues 37, 4, S. 769-787.

Beckert, Jens 2009. "The great transformation of embeddedness: Karl Polanyi and the new economic sociology ", in Market and society: the great transformation, hrsg. v. Hann, Chris; Hart, Keith, S. 38-55. New York: Cambridge University Press.

Beckert, Jens; Streeck, Wolfgang 2008. Economic sociology and political economy: a programmatic perspective. MPIfG Working Paper Nr. 08/4. Köln: Max-Planck-Institut für Gesellschaftsforschung.

Bell, Daniel 1976. The cultural contradictions of capitalism. New York: Basic Books.

Burawoy, Michael 2005. »2004 American Sociological Association Presidential address: for public sociology «, in American Sociological Review 70, 1, S. 4-28.

Calhoun, Craig; Derluguian, Georgi. Hrsg. 2011. Business as usual: the roots of the global financial meltdown. New York: Social Science Research Council and New York University Press.

40 Versammelt unter anderem in der Society for the Advancement of Socio-Economics (SASE) und um ihre Zeitschrift Socio-Economic Review.

Leviathan, 40. Jg., 1/2012 
Camic, Charles. Hrsg. 1991. Talcott Parsons: the early essays. Chicago: University of Chicago Press.

Etzioni, Amitai 1968. The active society. New York: The Free Press.

Fritz, Wolfgang; Mikl-Horke, Gertraude 2007. Rudolf Goldscheid: Finanzsoziologie und ethische Sozialwissenschaft. Wien: LIT Verlag.

Granovetter, Mark 1985. "Economic action and social structure: the problem of embeddedness ", in American Journal of Sociology 91, 4, S. 481-510.

Habermas, Jürgen 1973. Legitimationsprobleme im Spätkapitalismus. Frankfurt a. M.: Suhrkamp.

Hall, Peter A.; Soskice, David 2001. "An introduction to varieties of capitalism ", in Varieties of capitalism: the institutional foundations of comparative advantage, hrsg. v. Hall, Peter A.; Soskice, David, S. 1-68. Oxford: Oxford University Press.

Hochschild, Arlie Russell 1997. The time bind: when work becomes home and home becomes work. New York: Henry Holt \& Company.

Hochschild, Arlie Russell 2003. The commercialization of intimate life: notes from home and work. Berkeley, Los Angeles: University of California Press.

Ingham, Geoffrey 2004. The nature of money. Cambridge: Polity Press.

Kaufmann, Jean-Claude 2011. Sex@mour. Wie das Internet unser Liebesleben verändert, übersetzt v. Anke Beck. Konstanz: UVK.

Keynes, John Maynard 1963 [1930]. »Economic possibilities for our grandchildren ", in J. M. Keynes, essays in persuasion, S. 358-373. New York: W. W. Norton \& Co.

Keynes, John Maynard 2009 [1936]. Allgemeine Theorie der Beschäftigung, des Zinses und des Geldes. Berlin: Duncker \& Humblot.

Krippner, Greta R. 2011. Capitalizing on crisis: the political origins of the rise of finance. Cambridge: Harvard University Press.

Lange, Carolin Dorothee 2011. "Was wird man wohl noch sagen dürfen « - Oder: Was hat Thilo Sarrazin eigentlich mit dem Sturm und Drang zu tun? MPIfG Working Paper Nr. 11/9. Köln: Max-Planck-Institut für Gesellschaftsforschung.

Lockwood, David 1964. "Social integration and system integration «, in Explorations in social change, hrsg. v. Zollschan, George K.; Hirsch, Walter, S. 244-257. London: Houghton Mifflin.

Mannheim, Karl 1951. Freedom, power \& democratic planning. London: Routledge \& Kegan Paul.

Marshall, T. H. 1965 [1949]. "Citizenship and social class", in Class, citizenship and social development: essays by T. H. Marshall, S. 71-134. Garden City: Anchor Books.

Miegel, Meinhard 2010. Exit. Wohlstand ohne Wachstum. Berlin: Propyläen.

O'Connor, James 1973. The fiscal crisis of the state. New York: St. Martin's Press.

Offe, Claus 1972. Strukturprobleme des kapitalistischen Staates: Aufsätze zur politischen Soziologie. Frankfurt a. M.: Suhrkamp.

Offe, Claus 2008. "Governance: `Empty Signifier oder sozialwissenschaftliches Forschungsprogramm?", in Governance in einer sich wandelnden Welt. Politische Vierteljahresschrift, Sonderheft 41, hrsg. v. Schuppert, Gunnar Folke; Zürn, Michael, S. 61-76. Wiesbaden: VS Verlag für Sozialwissenschaften.

Polanyi, Karl 1957 [1944]. The great transformation: the political and economic origins of our time. Boston: Beacon Press.

Sarrazin, Thilo 2010. Deutschland schafft sich ab. Wie wir unser Land aufs Spiel setzen. München: Deutsche Verlags-Anstalt.

Schäfer, Armin 2010: "Die Folgen sozialer Ungleichheit für die Demokratie in Westeuropa «, in Zeitschrift für vergleichende Politikwissenschaft 4, 1, S. 131-156.

Schauer, Alexandra; van Dyk, Silke 2010. »Die DGS und der Nationalsozialismus ", in Soziologie 39,4, S. 411-424.

Stiglitz, Joseph E.; Sen, Amartya; Fitoussi, Jean-Paul 2009. Report by the Commission on the Measurement of Economic Performance and Social Progress, http://www.stiglitz-sen-fitous si.fr/documents/rapport_anglais.pdf (Zugriff vom 24.01.2012).

Streeck, Wolfgang 2009. Re-forming capitalism: institutional change in the German political economy. Oxford: Oxford University Press.

Streeck, Wolfgang 2011 a. »A crisis of democratic capitalism", in New Left Review 71, S. 1-25. 
Streeck, Wolfgang 2011 b. »Man weiß es nicht genau: Vom Nutzen der Sozialwissenschaften für die Politik ", in Wissenschaftliche Politikberatung, hrsg. v. Kraul, Margret; Stoll, Peter-Tobias, S. 15-41. Göttingen: Wallstein Verlag.

Streeck, Wolfgang 2011 c. »Taking capitalism seriously: towards an institutional approach to contemporary political economy «, in Socio-Economic Review 9, 1, S. 137-167.

Thelen, Kathleen 2002. "How institutions evolve: insights from comparative-historical analysis ", in Comparative historical analysis in the social sciences, hrsg. v. Mahoney, James; Rueschemeyer, Dietrich, S. 208-240. New York: Cambridge University Press.

Thelen, Kathleen 2004. How institutions evolve. The political economy of skills in Germany, Britain, the United States, and Japan. Cambridge: Cambridge University Press.

van Dyk, Silke; Schauer, Alexandra 2010. "...daß die offizielle Soziologie versagt hat «: Zur Soziologie im Nationalsozialismus, der Geschichte ibrer Aufarbeitung und der Rolle der DGS. Essen: Deutsche Gesellschaft für Soziologie.

Weber, Max 1895. Der Nationalstaat und die Volkswirtschaftspolitik. Freiburg i. Br., Leipzig: Mohr-Siebeck.

\section{Autor}

Prof. Dr. Wolfgang Streeck

Max-Planck-Institut für Gesellschaftsforschung

Paulstrasse 3

50676 Köln

Übersetzung: Luisa Seeling

Leviathan, 40. Jg., 1/2012 